Original Article

\title{
Effects of Concurrent Training Order on Follistatin, Physical Fitness Factors and Functional Capacity of Postmenopausal Women
}

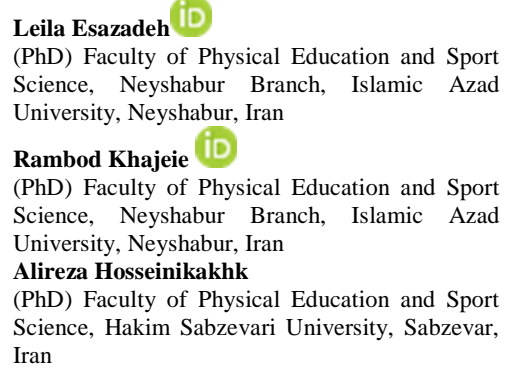

Corresponding author: Rambod Khajeie Email: R.khajeie@gmail.com

Tel: +989151141409

Address: Faculty of Physical Education and Sport Science, Neyshabur Branch, Islamic Azad University, Neyshabur, Iran

Received: $2019 / 07 / 20$

Revised: 2019/09/8

Accepted: 2019/09/8

\section{(cc) (i) \&}

This work is licensed under a Creative Commons Attribution 4.0 License.

DOI: $10.29252 / \mathrm{mlj} .14 .6 .28$

\begin{abstract}
Background and objectives: It has been suggested that concurrent training (CT) improves both health and performance during aging. However, possible effects of CT order have not been evaluated in menopausal women. Therefore, the aim of this study was to evaluate effects of CT order on follistatin, physical fitness factors and functional capacity of postmenopausal women.

Methods: In this semi-experimental study, 33 healthy, sedentary, postmenopausal women were divided into three groups of endurance training-resistance training $(\mathrm{ET}+\mathrm{RT} ; \mathrm{n}=11)$, resistance training-endurance training $(\mathrm{RT}+\mathrm{ET} ; \mathrm{n}=10)$ and control $(\mathrm{C} ; \mathrm{n}=12)$. The training exercises were performed three times a week for eight weeks.
\end{abstract}

Results: Weight and body fat percentage did not differ significantly between the groups $(\mathrm{P}>0.05)$. Muscular strength, endurance and aerobic capacity increased significantly in both training groups $(\mathrm{P}<0.05)$. However, there was no significant difference between the effects of the training orders. There was a significant time $\times$ group interaction regarding the follistatin levels after the CT intervention. However, there was no significant difference in the follistatin levels between the groups.

Conclusion: Based on the results, the order of eight weeks of CT training does not significantly affect body fat percentage, physical fitness factors and functional capacity of postmenopausal women. However, the percentage changes in the mentioned variables are higher in the ET+RT exercise order.

Keywords: Resistance Training, Endurance Training, Menopause, Body Composition. 


\section{INTRODUCTION}

The aging process in humans is accompanied with a progressive reduction in muscle mass, strength and function, which has a critical influence on individuals' abilities in performing daily activities (1). Women are more susceptible to cardiovascular disease compared to men due to a higher amount of adipose tissue (2). Reduction in the secretion of estrogen and progesterone during menopause leads to fat accumulation and osteoporosis and increases the risk of fractures, especially in the pelvic area (3). Oxidative stress, blood lipids and body fat percentage (BFP) after menopause have an important role in development of cardiovascular disease, hypertension, atherosclerosis, diabetes, cardiac hypertrophy and myocardial infarction (4). Physical inactivity is one of the factors that increases fat accumulation in the body, which results in increased risk of obesity and reduced sex hormones in postmenopausal women (5). Various physical activities have been suggested to cope with fat accumulation and to improve functional capacity in the aging process. The primary goals of resistance training (RT) are increased strength, muscular endurance, increased bone density and decreased blood pressure and BFP (6). Endurance training (ET) is another type of exercise that is used to increase maximum oxygen consumption and to change cardiac function and blood volume (7). Improvement of endothelial function and arterial stiffness and reduction of oxidative stress are other effects of these exercise interventions. The combination of RT and ET known as concurrent training (CT) can improve physical performance as well as body composition in older people (8). This strategy has greater benefits than RT or ET alone and is often used to promote fat loss, weight control, increased physical function and cardiovascular fitness (9).

Follistatin (FLST) is a myokine involved in the regulation of muscle mass and is a positive regulator of skeletal muscle mass. It has been shown that FLST is able to decrease biological effects of growth differentiation factor family, which positively increase skeletal muscle mass (10). It has been shown that FLST levels increase significantly after lower-body RT in trained volleyball players (11). Also, FLST levels increase significantly after combined lower body + upper body RT in middle-aged men (10). Some studies revealed that RT alone has a negative effect on ET benefits, but CT would have positive effects on cardiovascular adaptations as well as muscle mass, strength and power (12).

The question remains that whether RT and ET would interfere with their physiological effect. On the other hand, some studies have shown that CT has no effect on long-term adaptations, and the strength gains are equal in both training methods. In addition, it has been shown that CT can increase basal metabolic rate and help reduce fat mass. Considering the importance of body fat indices in metabolic syndrome and cardiovascular disease, we aimed to investigate the effects of CT orders on different physical fitness indicators and functional capacity of postmenopausal women. MATERIALS AND METHODS

In this semi-experimental study, 33 healthy, sedentary, postmenopausal women were divided into three groups of ET-RT (ET+RT; $\mathrm{n}=11)$, RT-ET (RT+ET; $\mathrm{n}=10)$ and control $(\mathrm{C}$; $\mathrm{n}=12$ ). Sample size was estimated by the $\mathrm{G}-$ Power software. The minimum sample size for three groups was 36 participants with respect to effect size of 0.27 , power of 0.8 and $\alpha=0.05$. Height and weight of the subjects were measured with precision of $1 \mathrm{~cm}$ and $0.1 \mathrm{~kg}$, respectively. Body mass index (BMI) was calculated by dividing body weight by height squared $\left(\mathrm{kg} / \mathrm{m}^{2}\right)$. Subjects who had any diseases such as diabetes or heart disease, musculoskeletal injury, history of smoking, history of drug or supplement consumption and history of exercise in the last six months were excluded from the study. During the study period, physical injury and irregular attendance at training sessions were considered as the exclusion criteria.

Both experimental groups performed the same RT and ET procedures. However, the order of the training was changed. The training interventions were carried out for eight weeks from low to high intensity according to overload principle. Both groups performed the exercises simultaneously after 15 minutes of warm-up. The RT+ET group first performed RT for 45 minutes, then after 10 minutes of rest, they performed ET for 20 to 40 minutes. The ET+RT group first performed ET for 20 to 40 minutes, then rested for 10 minutes and then performed RT for 45 minutes. Resistant training exercises included biceps curl, triceps 
pushdown, lat pulldown, lateral raise, incline chest press, leg extension, leg curl and calf raise. In the first two weeks, the exercises were performed three sessions per week. Between the third and eighth week, some upper and lower body exercises (biceps curl, triceps pushdown, incline chest press, leg extension and leg curl) were performed on Saturday and the remaining exercises were performed on Sunday.

Endurance training exercises (aerobic exercise i.e., lower and upper body exercises) were performed at $65 \%$ of maximum heart rate in the initial training sessions, and the exercise intensity reached $80 \%$ of maximum heart rate by the end of the training protocol. A polar heart rate monitor (Polar S810, Polar Electro, Kempele, Finland) was used to control the exercise intensity. The total ET exercise volume was 20 minutes in the initial training sessions and was increased to 40 minutes by the end of the training protocol, which was performed with four sets followed by a minute rest interval.

Leg extension exercise was used to determine lower body strength. The participants performed the test three times with 3-5 minutes rests between each attempt to reduce measurement error due to fatigue. All three attempts were recorded, while the highest was used for analysis. After the testing session, the participant's maximal strength was predicted using the following formula:

1 repetition maximum $(1 \mathrm{RM})=$ weight/ (1.0278-0.0278xreps).

After the lower body strength test, the participants used $50 \%$ of the calculated strength test until muscular fatigue. Then, the total number of attempts was multiplied by lifted weight recorded as lower body endurance. Body fat percentage was determined using a caliper device (Lafayette, model 01127 , USA) with an accuracy of $1 \mathrm{~mm}$. Three skinfolds were measured, namely above the iliac femoral neck and triceps brachia on the right side. Then, BFP was calculated by using the Jackson and Pollock formula (13).

Before blood sampling, the participants were asked to fast for 12 hours (an overnight fast) and abstain from physical activity for 36 hours. Blood samples $(5 \mathrm{~mL})$ were obtained from the cubital vein 48 hours before the first training session and 48 hours after the last training session. After clotting, blood samples were centrifuged at 3,000 RPM for 10 minutes. Spun serum was removed and frozen at $-80^{\circ} \mathrm{C}$ for analysis. Serum FLST (bound) concentrations were measured using enzymelinked immunosorbent assay kits (human follistatin, ZellBio $\mathrm{GmbH}$, Germany) according to the manufacturer's instructions. Normality of data was confirmed using the Shapiro-Wilk test. The difference between variables such as age, weight and BMI was evaluated by ANOVA. Statistical analysis of variance (repeated measures ANOVA) and Bonferroni post hoc test were used for data analysis. All statistical analyses were performed using SPSS software (version 22) at significance level of 0.05 .

Spun serum was removed and frozen at $-80^{\circ} \mathrm{C}$ for analysis. Serum FLST (bound) concentrations were measured using enzymelinked immunosorbent assay kits (human follistatin, ZellBio $\mathrm{GmbH}$, Germany) according to the manufacturer's instructions.

Normality of data was confirmed using the Shapiro-Wilk test. The difference between variables such as age, weight and BMI was evaluated by ANOVA. Statistical analysis of variance (repeated measures ANOVA) and Bonferroni post hoc test were used for data analysis. All statistical analyses were performed using SPSS software (version 22) at significance level of 0.05 .

\section{RESULTS}

At baseline, weight and BFP did not differ significantly between the groups. The effects of CT on the study variables are presented in table 1. Main effect, time effect and interaction effect of all variables are presented in table 2 . The time $\times$ group interaction regarding weight and BFP was not statistically significant. However, this was significant regarding leg extension for strength and endurance as well as the 6-minute walking test $(\mathrm{P}<0.05)$. The Bonferroni post hoc revealed a significant increase in bench press strength and endurance. However, we saw a significant decrease in the control group. The 6-minute walking test values significantly increased in both experimental groups. Percent changes were more profound in the ET+RT group for all variables. There was no significant time $x$ group interaction regarding the FLST levels after the CT intervention. In addition, there was no significant difference in the FLST levels between the study groups. 
Table 1. Pre- and post-intervention values of the study variables following eight weeks of concurrent training

\begin{tabular}{|c|c|c|c|c|c|c|c|c|c|}
\hline \multirow[t]{2}{*}{ Group } & \multicolumn{3}{|c|}{ Endurance training + resistance training } & \multicolumn{3}{|c|}{ Resistance training + endurance training } & \multicolumn{3}{|c|}{ Control } \\
\hline & $\begin{array}{c}\text { Pre- } \\
\text { intervention }\end{array}$ & $\begin{array}{c}\text { Post- } \\
\text { intervention }\end{array}$ & Changes & $\begin{array}{c}\text { Pre- } \\
\text { intervention }\end{array}$ & $\begin{array}{c}\text { Post- } \\
\text { intervention }\end{array}$ & Changes & $\begin{array}{c}\text { Pre- } \\
\text { intervention }\end{array}$ & $\begin{array}{c}\text { Post- } \\
\text { intervention }\end{array}$ & Changes \\
\hline Weight (kg) & $66.6 \pm 9.4$ & $65.3 \pm 8.5$ & -0.01 & $70 \pm 8.4$ & $69.8 \pm 8.1$ & -0.009 & $70.8 \pm 11.5$ & $70.7 \pm 10.4$ & -0.001 \\
\hline BFP $(\%)$ & $39.8 \pm 7.5$ & $35.6 \pm 4.12$ & -0.1 & $38.9 \pm 2.8$ & $38.3 \pm 1.7$ & -0.01 & $36.9 \pm 4.4$ & $36.8 \pm 3.9$ & -0.003 \\
\hline Follistatin & $10.1 \pm 1.6$ & $9.86 \pm 2.04$ & -0.028 & $8.72 \pm 2.94$ & $7.68 \pm 2.79$ & -0.119 & $8.81 \pm 2.63$ & $8.58 \pm 2.08$ & -0.026 \\
\hline $\begin{array}{l}\text { 6-minute } \\
\text { walking } \\
(\mathrm{m})\end{array}$ & $644.9 \pm 80.8$ & $721.5 \pm 71.4$ & 0.1 & $664.2 \pm 32.9$ & $715.6 \pm 84.7$ & 0.07 & $626.6 \pm 111.1$ & $645 \pm 128.4$ & 0.02 \\
\hline $\begin{array}{c}\text { Lower limb } \\
\text { strength } \\
\text { (kg) }\end{array}$ & $29 \pm 11.5$ & $56 \pm 17$ & 0.9 & $40.5 \pm 12.1$ & $57 \pm 11.5$ & 0.4 & $43.3 \pm 13.2$ & $45.4 \pm 15.5$ & 0.04 \\
\hline $\begin{array}{c}\text { Lower limb } \\
\text { endurance } \\
(\mathbf{R} \times \mathbf{k g})\end{array}$ & $262.7 \pm 121.5$ & $397.9 \pm 144.2$ & 0.5 & $328.7 \pm 160.7$ & $437.5 \pm 193$ & 0.3 & $328.3 \pm 138.8$ & $335.2 \pm 174$ & 0.02 \\
\hline
\end{tabular}

Table 2. Effects of the eight-week concurrent training exercises on the research variables analyzed by repeated measures ANOVA

\begin{tabular}{|c|c|c|c|c|c|c|c|c|c|}
\hline \multirow[b]{2}{*}{ Variable } & \multicolumn{3}{|c|}{ Group effect } & \multicolumn{3}{|c|}{ Time effect } & \multicolumn{3}{|c|}{ Interaction effect (Time $\times$ Group) } \\
\hline & $\bar{F}$ & $\mathrm{P}-\mathrm{V}$ & $\bar{d}$ & $\bar{F}$ & $\mathrm{P}-\mathrm{v}$ & $\bar{d}$ & $\bar{F}$ & $\mathrm{P}-\mathrm{v}$ & $\bar{d}$ \\
\hline Weight (kg) & 0.8 & 0.4 & 0.05 & 3.1 & 0.08 & 0.09 & 1.79 & 0.18 & 0.1 \\
\hline BFP $(\%)$ & 0.506 & 0.6 & 0.033 & 5.03 & 0.032 & 0.144 & 3.302 & 0.051 & 0.18 \\
\hline Follistatin & 0.163 & 1.93 & 0.11 & 0.122 & 2.53 & 0.07 & 0.549 & 0.611 & 0.03 \\
\hline $\begin{array}{l}\text { 6-minutes walking } \\
\text { (m) }\end{array}$ & 1.23 & 0.3 & 0.07 & 44.55 & 0.000 & 0.59 & 5.61 & 0.008 & 0.27 \\
\hline $\begin{array}{l}\text { Lower Limb } \\
\text { strength (kg) }\end{array}$ & 0.566 & 0.5 & 0.036 & 165.06 & 0.000 & 0.846 & 39.61 & 0.000 & 0.725 \\
\hline $\begin{array}{c}\text { Lower limb } \\
\text { endurance }(\mathbf{R} \times \mathbf{k g})\end{array}$ & 0.419 & 0.6 & 0.027 & 29.60 & 0.000 & 0.497 & 6.83 & 0.004 & 0.313 \\
\hline
\end{tabular}




\section{DISCUSSION}

This study investigated the effects of eight weeks of different CT orders on physical fitness, functional capacity and body composition factors of postmenopausal women. The results of this study showed that CT order had no significant impact on the variables. Weight and BFP changes were not significant in all three groups. Previous research on the effects of CT exercises on BFP is very limited and contradictory. Inconsistent with our findings, previous studies showed a significant decrease in the BFP compared to the control group in both CT orders, although there was no difference between the groups $(14,15)$.

BFP may change following CT exercise if the energy input is less than the energy consumed (16). Some studies reported that CT exercise significantly reduced BFP compared to other types of exercises but not compared to different exercise orders (17). Eddens et al. concluded that CT did not have a significant effect on BFP (18). In addition, Davitt et al. reported that ET performed before or after RT had the same effect on body composition (19). According to Alves et al., the order of CT does not affect the energy consumption (20), which is line with our findings. It has been suggested that CT with different orders can promote weight loss by reducing BFP in obese men, although the RT+ET sequence has better effects (21). However, we found that the effects of ET+RT was more notable compared to the RT+ET order. This may be due to the difference in the type of ET protocol used in the two studies. In the present study, strength and endurance of the lower limb muscles increased significantly in both experimental groups. However, there was no significant difference between the two groups in terms of strength and endurance of the lower limb muscles. Although FLST levels decreased in both experimental groups, this change was not statistically significant. Consistent with our study, Karatrantou et al. did not observe any significant change in muscular strength and endurance (22). However, this finding is inconsistent with findings of some previous studies $(24,25)$. Two other studies also reported that $\mathrm{CT}$ order does not affect strength gain $(23,24)$. It has been proposed that the effect of exercise order strongly relies on the type of ET or RT in a way that after highintensity ET, the power production decreases, while low-intensity ET less likely contributes to fatigue (25). Some studies pointed out that by adding ET stimulus to RT, fatigue reduces muscular adaptation (26) and the level of intracellular glycogen (27). In addition, maximal muscle strength would be decreased due to a reduction in neural and peripheral contraction mechanisms (28). It is supposed that when ET is performed immediately before RT, total acute and systematic fatigue interferes with long-term RT (29).

In our study, aerobic capacity did not differ significantly between the two exercise groups, but the percentage changes in the ET+RT group was higher. Researchers argue that simultaneous combined exercises can create long-term adaptations compared to a single exercise type $(30,31)$.

Our findings showed that CT exercise with different orders had no significant effect on BFP in postmenopausal women. The volume and intensity of ET and RT, the number of sets, the number of repetitions per set and tempo of the exercises are critical factors that may contribute to decreased BFP and increased muscular hypertrophy. Since menopause is associated with cardiovascular risk factors and metabolic disorders (32), reduced sex hormones, fat accumulation and reduced body mass may be evident in menopausal women (4). In postmenopausal women, physical activity has a significant effect on the physical activity outcomes and BFP changes. The results of the present study suggest that there is no significant difference in blood and physical fitness factors between the CT orders. Therefore, both CT exercise orders can be performed interchangeably to increase strength and muscular endurance.

The present study had some limitations including the lack of precise monitoring of the participants' diet and daily activities.

\section{CONCLUSION}

Based on the results, the order of eight weeks of CT training does not significantly affect BFP, physical fitness factors and functional capacity in postmenopausal women. However, the percent changes in the mentioned variables are higher in the ET+RT exercise order.

\section{ACKNOWLEDGMENTS}

The authors are grateful to all participants.

\section{CONFLICT OF INTEREST}

The authors declare that there is no conflict of interest. 


\section{REFERENCES}

1.Van Kan GA. Epidemiology and consequences of sarcopenia. JNHA-The Journal of Nutrition, Health and Aging. 2009; 13(8): 708-12.

2.Zaros PR, Pires CER, Bacci M, Moraes C, Zanesco A. Effect of 6-months of physical exercise on the nitrate/nitrite levels in hypertensive postmenopausal women. BMC women's health. 2009; 9(1): 17.

3. Organization WH. Research on the menopause in the 1990s: report of a WHO scientific group. 1996.

4.Crist BL, Alekel DL, Ritland LM, Hanson LN, Genschel U, Reddy MB. Association of oxidative stress, iron, and centralized fat mass in healthy postmenopausal women. Journal of Women's Health. 2009;18(6):795-801.

5.Krishnan K, Bassett JK, MacInnis RJ, English DR, Hopper JL, McLean CA, et al. Associations between weight in early adulthood, change in weight and breast cancer risk in postmenopausal women. Cancer Epidemiology and Prevention Biomarkers. 2013:cebp. 0136.2013.

6.Wood RH, Reyes R, Welsch MA, Favaloro-Sabatier J, Sabatier M, Lee CM, et al. Concurrent cardiovascular and resistance training in healthy older adults. Medicine \& Science in Sports \& Exercise. 2001; 33(10): 1751-8.

7.McNeil J, Brenner D, Courneya K, Friedenreich C. Doseresponse effects of aerobic exercise on energy compensation in postmenopausal women: combined results from two randomized controlled trials. International Journal of Obesity. 2017; 41(8): 1196.

8.Moghadam B, Bagheri R, Ashtary-Larky D, Tinsley G, Eskandari M, Wong A, et al. The Effects of Concurrent Training Order on Satellite Cell-Related Markers, Body Composition, Muscular and Cardiorespiratory Fitness in Older Men with Sarcopenia. The journal of nutrition, health \& aging. 2020; 24(7): 796-804.

9.Takeshima N, Rogers ME, Islam MM, Yamauchi T, Watanabe $\mathrm{E}$, Okada A. Effect of concurrent aerobic and resistance circuit exercise training on fitness in older adults. European journal of applied physiology. 2004; 93(1-2): 173-82.

10.Bagheri R, Rashidlamir A, Motevalli MS, Elliott BT, Mehrabani J, Wong A. Effects of upper-body, lower-body, or combined resistance training on the ratio of follistatin and myostatin in middle-aged men. European journal of applied physiology. 2019: 1-11.

11.Bagheri R, Rashidlamir A, Attarzadeh Hosseini SR. effect of Resistance Training with Blood Flow Restriction onfollistatin to myostatin Ratio, Body Composition and Anaerobic Power of Trained-Volleyball Players. Medical Laboratory Journal. 2018; 12 .

12.Schoenfeld B. Science and Development of Muscle Hypertrophy: Human Kinetics; 2016.

13.Eston R, Reilly $\mathrm{T}$. Kinanthropometry and exercise physiology laboratory manual: tests, procedures and data: volume two: physiology: Routledge; 2013.

14.Faramarzi M, Bagheri L, Banitalebi E. Effect of sequence order of combined strength and endurance training on new adiposity indices in overweight elderly women. Isokinetics and Exercise Science. 2018(Preprint): 1-9.

15.Cadore EL, Izquierdo M, Pinto SS, Alberton CL, Pinto RS, Baroni BM, et al. Neuromuscular adaptations to concurrent training in the elderly: effects of intrasession exercise sequence. Age. 2013; 35(3): 891-903.

16.Dolezal BA, Potteiger JA. Concurrent resistance and endurance training influence basal metabolic rate in nondieting individuals. Journal of applied physiology. 1998; 85(2): 695700 .
17.Hu G, Tuomilehto J, Silventoinen K, Barengo N, Jousilahti P. Joint effects of physical activity, body mass index, waist circumference and waist-to-hip ratio with the risk of cardiovascular disease among middle-aged Finnish men and women. European heart journal. 2004;25(24):2212-9.

18.Eddens L, van Someren K, Howatson G. The role of intrasession exercise sequence in the interference effect: a systematic review with meta-analysis. Sports Medicine. 2018;48(1):177-88. 19.Davitt PM, Pellegrino JK, Schanzer JR, Tjionas H, Arent SM. The effects of a combined resistance training and endurance exercise program in inactive college female subjects: Does order matter? The Journal of Strength \& Conditioning Research. 2014; 28(7): 1937-45.

20.Alves J, Saavedra F, Simão R, Novaes J, Rhea MR, Green D, et al. Does aerobic and strength exercise sequence in the same session affect the oxygen uptake during and postexercise? The Journal of Strength \& Conditioning Research. 2012;26(7):18728 .

21.Sheikholeslami-Vatani D, Siahkouhian M, Hakimi M, AliMohammadi M. The effect of concurrent training order on hormonal responses and body composition in obese men. Science \& Sports. 2015;30(6):335-41.

22.Karatrantou K, Gerodimos V, Häkkinen K, Zafeiridis A. Health-Promoting Effects of Serial vs. Integrated Combined Strength and Aerobic Training. International journal of sports medicine. 2017;38(01):55-64.

23.Chtara M, Chaouachi A, Levin GT, Chaouachi M, Chamari $\mathrm{K}$, Amri M, et al. Effect of concurrent endurance and circuit resistance training sequence on muscular strength and power development. The Journal of Strength \& Conditioning Research. 2008;22(4):1037-45.

24.Collins MA, Snow TK. Are adaptations to combined endurance and strength training affected by the sequence of training? Journal of sports sciences. 1993;11(6):485-91.

25.Fyfe JJ, Bishop DJ, Stepto NK. Interference between concurrent resistance and endurance exercise: molecular bases and the role of individual training variables. Sports medicine. 2014; 44(6): 743-62.

26.Hickson RC. Interference of strength development by simultaneously training for strength and endurance. European journal of applied physiology and occupational physiology. 1980; 45(2-3): 255-63.

27.Jacobs I, Kaiser P, Tesch P. Muscle strength and fatigue after selective glycogen depletion in human skeletal muscle fibers. European Journal of Applied Physiology and Occupational Physiology. 1981; 46(1): 47-53.

28.Lepers R, Hausswirth C, Maffiuletti N, Brisswalter J, Van Hoecke J. Evidence of neuromuscular fatigue after prolonged cycling exercise. Medicine \& Science in Sports \& Exercise. 2000; 32(11): 1880-6.

29. Mitchell WK, Atherton PJ, Williams J, Larvin M, Lund JN, Narici M. Sarcopenia, dynapenia, and the impact of advancing age on human skeletal muscle size and strength; a quantitative review. Frontiers in physiology. 2012; 3: 260.

30.Dudley GA, Djamil R. Incompatibility of endurance-and strength-training modes of exercise. Journal of Applied Physiology. 1985; 59(5): 1446-51.

31.Glowacki SP, Martin SE, Maurer A, Baek W, Green JS, Crouse SF. Effects of resistance, endurance, and concurrent exercise on training outcomes in men. Medicine and science in sports and exercise. 2004; 36(12): 2119-27.

32.Garcia M, Mulvagh SL, Bairey Merz CN, Buring JE, Manson JE. Cardiovascular disease in women: clinical perspectives. Circulation research. 2016; 118(8): 1273-93.

\section{How to Cite:}

Esazadeh L, Khajeie R, Hosseinikakhk A . [Effects of Concurrent Training Order on Follistatin, Physical Fitness Factors and Functional Capacity of Postmenopausal Women]. mljgoums. 2020; 14(6): 28-33. DOI: 10.29252/mlj.14.6.28 